\title{
Jet breakup in superfluid and normal liquid ${ }^{4} \mathrm{He}$
}

\author{
N. B. Speirs $\odot,{ }^{1, *}$ K. R. Langley $\odot,{ }^{1}$ P. Taborek $\odot,{ }^{2}$ and S. T. Thoroddsen $\oplus^{1}$ \\ ${ }^{1}$ Division of Physical Sciences and Engineering, King Abdullah University of Science and Technology \\ (KAUST), Thuwal 23955-6900, Saudi Arabia \\ ${ }^{2}$ Department of Physics and Astronomy, University of California Irvine, Irvine, California 92697, USA
}

(Received 7 October 2019; accepted 4 March 2020; published 2 April 2020)

\begin{abstract}
Past studies have shown that liquid jet breakup behavior can be classified into five regimes: Rayleigh, first wind, sinuous, second wind, and atomization. By experimentally examining the breakup of superfluid and normal liquid ${ }^{4} \mathrm{He}$ in an atmosphere of its own vapor, we investigate the evolution of the jet behavior over a large range of the traditional three-dimensional parameter space of the Ohnesorge number $\left[\mathrm{Oh}_{l} \sim O\left(10^{-5}-10^{-2}\right)\right]$, Reynolds number $\left[\operatorname{Re}_{l} \sim O\left(10^{2}-10^{6}\right)\right]$, and gas-liquid density ratio $\left[\rho_{g} / \rho_{l} \sim O\left(10^{-4}-1\right)\right]$. Using dimensional analysis we find that the transition from Rayleigh to first-wind breakup occurs at a constant liquid Weber number, and that the transitions from first wind to sinuous, and sinuous to second wind occur at constant gas Weber numbers. The proposed transitions, which differ from some previous studies, are well supported by our new experimental data that extend over all three dimensions of the parameter space. We do not observe any obvious effects of superfluidity on the breakup behavior. In addition, we examine the breakup length and comment on the transition of a liquid jet to a gaseous jet as the temperature passes through the critical point.
\end{abstract}

DOI: 10.1103/PhysRevFluids.5.044001

\section{INTRODUCTION}

When one fluid exits a circular nozzle into another immiscible fluid it forms an unstable cylindrical structure [1]. Various forces acting on the interface cause the jet to break up into a stream of droplets. The appearance of jets undergoing breakup varies significantly depending on the input parameters, with variations in the down-stream breakup position, the resulting droplet size, and angular spread of the stream, as shown in Fig. 1. This paper investigates the breakup of jets of superfluid and normal liquid helium four, ${ }^{4} \mathrm{He}$, as it exits a nozzle into its own vapor. After reviewing the previous jet breakup studies we find some discrepancies in the literature on the different breakup modes or regimes and the parameter space for which they occur. Hence, we focus on more fully describing the breakup behaviors and the transitions between them. We also comment on the jet breakup length from the nozzle and the breakup of jets near the critical point.

Plateau theoretically studied the stability of an infinite cylinder of liquid and found it to exist in an unstable equilibrium [1]. With his mathematical approach, he found that any perturbation with wavelength $\lambda$ greater than the circumference of the cylinder, $2 \pi R_{j}$, where $R_{j}$ is the jet radius, is unstable and allows surface tension to break up the cylinder into droplets, decreasing the surface

*nathan.speirs@kaust.edu.sa

Published by the American Physical Society under the terms of the Creative Commons Attribution 4.0 International license. Further distribution of this work must maintain attribution to the author(s) and the published article's title, journal citation, and DOI. 

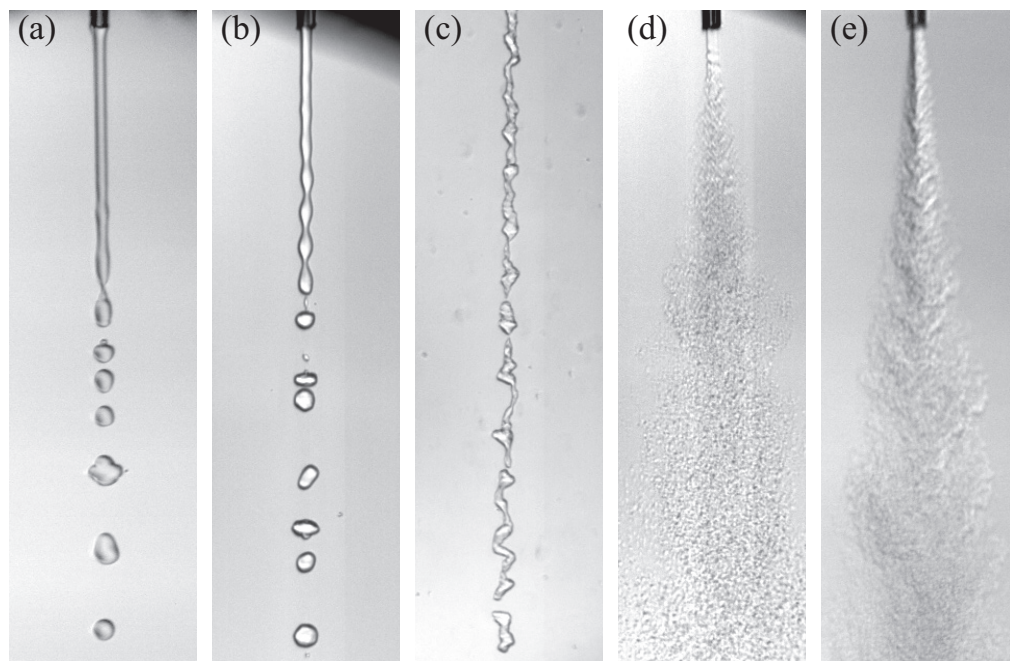

FIG. 1. A cylindrical jet of liquid ${ }^{4} \mathrm{He}$ emanates from a nozzle with inner diameter $52 \mu \mathrm{m}$ and breaks up into a stream of droplets or a spray with various characteristics and appearances for differing input parameters. (a) Rayleigh breakup occurs when droplets pinch off near the bottom tip. (b) first-wind breakup occurs at higher velocities and appears similar to Rayleigh breakup, but in this case the jet pinches together at multiple adjacent locations in rapid succession. (c) In sinuous breakup the jet buckles forming a twisting or corkscrewlike structure. (d) Second-wind and (e) atomization breakups occur at the highest velocities, in which the jet fragments into a mist of very small droplets that forms a conical shape. When this breakup occurs downstream of the nozzle (d) it is known as second wind and when breakup begins within about one diameter from the nozzle (e) the name changes to atomization.

area and hence the surface energy of the liquid. Rayleigh expanded Plateau's study showing for an inviscid liquid that the most unstable and fastest growing mode occurs at wavelength $\lambda=9.01 R_{j}$ [2-4]. This basic unstable nature of a liquid cylinder later became known as the Rayleigh-Plateau instability and served as the basis for understanding the various forces acting on free-surface jets.

The forces counteracting the inertia of a free-surface jet include: surface tension, aerodynamic forces or Bernoulli pressures, and viscous forces [5,6]. Varying the relative quantities of these forces changes the appearance and nature of the breakup as shown in Fig. 1. When the velocity and viscosity of the jet are low, surface tension forces induce the pinch off of droplets near the bottom tip of the jet leading to an axisymmetric breakup as shown in Fig. 1(a). This is called Rayleigh breakup. Haenlein [5] explains that as the jet velocity $U_{j}$ increases the surrounding gas begins to have an effect. As the gas flows over the undulating surface of the jet its velocity increases over the peaks and decreases in the troughs. This induces Bernoulli pressures that are low at the peaks and high in the troughs which accentuate the influence of surface tension causing the jet to pinch off more quickly. Reitz named this type of breakup, shown in Fig. 1(b), first wind, emphasizing the first influence of aerodynamic effects as the velocity increases [6]. As the aerodynamic forces increase further, they act on the asymmetric disturbances of the jet, pushing it out of symmetry and bending it into a wave shape against the force of surface tension [5,7-9] [Fig. 1(c)]. Weber [10], Debye and Daen [11], and Entov and Yarin [7-9,12] further investigate this bending instability and breakup with linear and nonlinear stability theories. This breakup mode has been called wavy $[5,10]$, screw symmetric or screwlike [13], bending [9], and sinuous [14-17], which is what we call it in this study. With even higher aerodynamic forces, short-wavelength, unstable waves grow on the jet surface breaking off as small droplets at the periphery and decreasing the jet diameter until the breakup reaches the axis $[18,19]$. This forms a conical spray of droplets with diameters much smaller than the jet diameter as shown in Fig. 1(d). This regime was named second wind by Reitz [6]. When the 


\begin{tabular}{|c|c|c|c|c|c|c|c|c|c|}
\hline & Rayleigh & transition & $1^{\text {st }}$ wind & transition & Sinuous & transition & $2^{\text {nd }}$ wind & transition & Atomization \\
\hline Haenlein (1932) [5] & $\begin{array}{l}\text { drop formation } \\
\text { without the }\end{array}$ & - & $\begin{array}{l}\text { drop formation } \\
\text { with the influence }\end{array}$ & - & $\begin{array}{l}\text { wave formation } \\
\text { due to the action }\end{array}$ & - & \multicolumn{3}{|c|}{ complete disintegration of the jet } \\
\hline Lee \& Spencer (1933) [23] & $\begin{array}{l}\text { rotationally } \\
\text { symmetric distur- } \\
\text { bances without } \\
\text { air influence }\end{array}$ & - & $\begin{array}{c}\text { rotationally } \\
\text { symmetric } \\
\text { disturbances with } \\
\text { air influence }\end{array}$ & - & wave formation & - & - & - & \\
\hline Ohnesorge (1936) [13] & axisy & mmetric perturb & ations & $\begin{array}{l}O h_{i}=125 R_{e}^{-54}[26] \\
\operatorname{Re}_{t}=460 h_{i}^{45}[16]\end{array}$ & screw symmetric & $\begin{array}{c}O h_{l}=2000 R e_{i}^{-43} \\
R e_{l}=270 O h_{i}^{445}[16\end{array}$ & & atomization & \\
\hline Miesse (1955) [24] & axisy & mmetric perturb & ations & - & screw symmetric & $\begin{array}{c}O h_{i}=100 R e_{i}^{.092} \\
\quad \text { or } \\
W_{e}=80.6\end{array}$ & & atomization & \\
\hline Ranz (1956) [15] & & yleigh or colum & & $W e_{E}=0.4$ & sinuous & $W_{e g}=13$ & & atomization & \\
\hline $\begin{array}{l}\text { Reviews of Reitz et al. } \\
(1978-1998)[6,18,27,28]\end{array}$ & Rayleigh & $\begin{array}{c}W_{e_{\mathrm{g}}}=1.2+3.41 O h_{i} \\
\text { [31] } \\
\text { or } \\
W_{e_{r}}=0.4[15] \\
\end{array}$ & & 1st wind & & $W e_{g}=13[15]$ & 2nd wind & 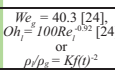 & atomization \\
\hline Saito et al. (2017) [19] & & varicose & & $O h_{f}=2.8 R e_{f}^{-J}$ & sinuous & $O h_{i}=22 R e_{t}^{-t}$ & & atomization & \\
\hline
\end{tabular}

FIG. 2. A summary of the literature discussing the jet breakup regimes is shown, emphasizing the various regime names, proposed transitions, and regime combinations. The regime names used in this paper are shown at the top of each of the five colored columns in order of increasing velocity from left to right. Below these are the names or descriptions used in each separate study. The white columns between regimes list any proposed transition criteria. Black, horizontal bars indicate that the authors combined regimes. Ohnesorge [13] did not provide quantitative transition criteria with his regime diagram, but others fit equations to his data and their fits are shown. Reitz et al. $[6,18,27,28]$ interpreted the findings of others in their reviews and the original source of their equations are also noted.

jet breaks up in a similar manner but at the nozzle exit, as shown in Fig. 1(e), the breakup is called atomization. It is assumed that some other force must speed up the breakup process in this regime as aerodynamic forces would require some jet length to take effect. Other potential breakup mechanism include some combination of pipe turbulence, velocity profile rearrangement, and cavitation [6]. In contrast to increasing aerodynamic effects, increasing viscosity tends to decrease the rate of breakup and results in the formation of larger droplets [20-22].

Ohnesorge was the first to show that the different regimes of jet breakup group together into specific locations on a regime diagram [13]. Yet the distinction and transition criteria between the five regimes discussed above is quite convoluted in the literature with no single paper discussing all five. Some confusion may have occurred due to the inconsistent naming of regimes as discussed by Reitz [6], publishing in both English [2-4,15,23,24] and German [10,13,25], and low initial appreciation of some works with later rediscovery as discussed by McKinley and Renardy [26]. To ease our discussion of the findings in the literature we have made Fig. 2 to help clarify the changing regime names, combinations or omissions, and various quantitative transition criteria found in the literature.

Haenlein took some of the initial photographic evidence of different jet behaviors, showed that four breakup regimes exist, and qualitatively discussed the forces causing the differences [5]. He described the four regimes as "drop formation without the influence of air" (Rayleigh), "drop formation with the influence of air" (first wind), "wave formation due to the action of air" (sinuous), and "complete disintegration of the jet" (a combination of second wind and atomization). Lee and Spencer [23] quickly followed presenting higher resolution photos of three of the same regimes which have been reproduced in several studies $[6,18,27]$ as examples of what each breakup mode looks like. Neither paper presented quantitative criteria for transition between regimes. Ohnesorge came next, discussing three breakup regimes, which he described as breakup by axisymmetric perturbations [Rayleigh and first wind, Figs. 1(a) and 1(b)], screw symmetric (sinuous), and atomization (second wind and atomization). Ohnesorge showed that straight lines on a log-log plot of the Reynolds and later-named Ohnesorge numbers divide the regimes but did not give equations for the transition lines he showed. After his paper, others have used his regime diagram to produce equations for these transitions $[14,16,26]$ or have modified his transition lines due to findings from their own data [24] as shown in Fig. 2. 
Ranz [15] took a little different approach to defining the regime transitions. He used characteristic pressure terms in an attempt to quantitatively define and explain the regime transitions (also reviewed and shown more clearly in Ref. [6]). He approximated the aerodynamic or inertial stress as $\rho U_{j}^{2} / 2$, the interfacial stress as $4 \sigma / d_{j}$ or $2 \sigma / d_{j}$ depending on $2 \mathrm{D}$ or $3 \mathrm{D}$ geometry, and the shear stress as $2 \mu U_{j} / d_{j}$, where $U_{j}$ is the jet velocity, $d_{j}$ is the jet diameter, $\sigma$ is the surface tension coefficient, $\rho$ is the fluid density, and $\mu$ is the dynamic viscosity. Subscripts of $g$ or $l$ indicate properties of the gas or liquid phases respectively and specify which phase applies the stress. The ratios of these stresses yield the liquid Reynolds number $\operatorname{Re}_{l}=\rho_{l} U_{j} d_{j} / \mu_{l}$, the liquid Weber number $\mathrm{We}_{l}=\rho_{l} U_{j}^{2} d_{j} / \sigma$, the gas Weber number $\mathrm{We}_{g}=\rho_{g} U_{j}^{2} d_{j} / \sigma$, the liquid Ohnesorge number $\mathrm{Oh}_{l}=\mathrm{We}_{l}^{1 / 2} / \operatorname{Re}_{l}=\mu_{l} /\left(\rho_{l} \sigma d_{j}\right)^{1 / 2}$, and the density ratio $\rho_{g} / \rho_{l}$. Using these terms Ranz [15] theorized that a dripping nozzle should form a columnar jet when the liquid inertia equals the interfacial pressure $\rho_{l} U_{j}^{2} / 2=4 \sigma / d_{j}$, which occurs when $\mathrm{We}_{l}=8$. Others [29,30] have studied the dripping to jetting transitions in much more detail finding it to be more complicated than a simple dependence on the $\mathrm{We}_{l}$ alone. He further said that Rayleigh or "columnar" breakup (this appears to include first wind) transitions to sinuous when the gas inertia equals one-tenth the interfacial stress $\rho_{g} U_{j}^{2} / 2=\frac{1}{10}\left(2 \sigma / d_{j}\right)$ or $\mathrm{We}_{g}=0.4$. Finally, he states that sinuous transitions to atomization (including second wind and atomization) when the gas inertia is of the same order as the interfacial pressure, which he defines as $\mathrm{We}_{g}=13$.

Reitz and coauthors later introduced their interpretation of the breakup regimes and boundaries in four separate, influential review papers $[6,18,27,28]$ (see Fig. 2). They discuss the Rayleigh, first wind, second wind, and atomization regimes, but omit the sinuous regime from their discussions, possibly combining it with first wind. They explain the regime boundaries through interpretation of others' works, resulting in applying the same transition criteria to different transitions. Citing Ranz [15] they state that Rayleigh jets form when $\mathrm{We}_{l}>8$ and transition to first wind when $\mathrm{We}_{g}=0.4$. They also provide an alternate Rayleigh-to-first-wind transition criteria as the point when the jet breakup length ceases to increase linearly with the jet velocity and cite Sterling and Sleicher [31] who found this to occur when $\mathrm{We}_{g}=1.2+3.41 \mathrm{Oh}_{l}^{0.9}$. Further interpreting Ranz's results, they state that first wind transitions to second wind when $\mathrm{We}_{g}=13$ showing they may have combined first wind and sinuous into one regime. Finally, they provide three alternate formulas for the transition from second wind to atomization: $\mathrm{We}_{g}=40.3$ citing Miesse [24] (it appears that a factor of 2 was lost because Miesse's transition converts to $\left.\mathrm{We}_{g}=80.6\right), \mathrm{Oh}_{l}=100 \mathrm{Re}_{l}^{-0.92}$ citing Miesse again, and $\rho_{l} / \rho_{g}=K f(T)^{-2}$, where $K$ is a function of the nozzle design and $f(T)$ is a function of $\mathrm{We}_{l}, \mathrm{Re}_{l}$, and $\rho_{l} / \rho_{g}$. One recent paper by Saito et al. [17] investigates a liquid jet injected into another immiscible liquid and discusses the transitions between Rayleigh, sinuous, and atomization as shown in Fig. 2. We discuss this paper more in Sec. IV.

The body of research on free surface jets also includes many other facets aside from the breakup regimes and transitions between them. Several authors investigate the intricacies of the jet breakup length from the nozzle showing effects from the mean velocity, velocity profile, transition to turbulence, and the ambient medium [18,32]. Others have investigated the size of the droplets formed from the breakup process [15], including the effect of the regime transitions [17], and extremely high ambient gas velocities such as when a jet ejects from the rear of an airplane [14]. McCarthy and Molloy [33] review the importance of nozzle design, discussing how the length to diameter ratio affects the jet's initial velocity profile and subsequently the surface roughness and other features. Contracting liquid filaments breakup in a similar manner to Rayleigh jets, forming one to multiple droplets of various sizes depending on $\mathrm{Oh}_{l}$ and the aspect ratio [22,34-37]. Other topics include: the forced breakup from sinusoidal perturbations [38-40], inkjet printing [41], liquid jets with surrounding coaxial gas flow [42], computer modeling [43,44], jet impact on solids [15,45] and liquids [46-49], flash boiling breakup when a jet injects into an atmosphere below its own vapor pressure [50,51], cooling and fragmentation of molten metal jets [52,53], and applications such as fuel injection [54,55] and other technologies [56]. At sufficiently low flow rates, jets do not form resulting in only dripping, which has also been extensively studied [57-61] including the transition from dripping to jetting $[29,30]$. 
(a)

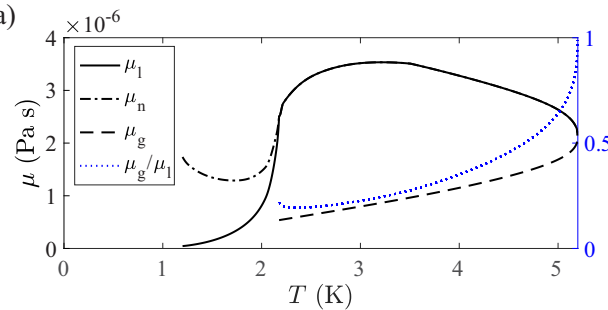

(c)

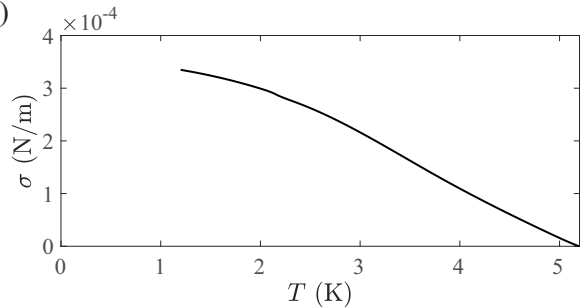

(b)

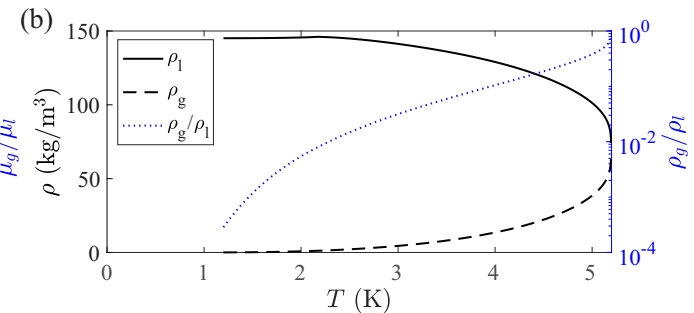

(d)

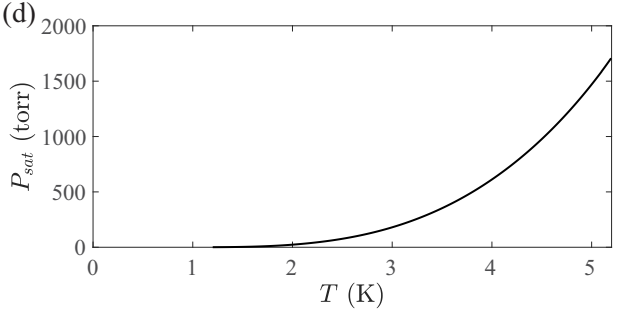

FIG. 3. The fluid properties along the saturation curve of gaseous and liquid ${ }^{4} \mathrm{He}$ are shown as a function of temperature $T$. Panel (a) shows the dynamic viscosity $\mu$ of the two phases separately, with the viscosity ratio shown on the right-hand $y$ axis. Below the $\lambda$ point $(T=2.17 \mathrm{~K})$ the liquid viscosity can be defined in two ways, as the viscosity of the normal component $\mu_{n}$, shown by the black dash-dotted line, or the effective total viscosity $\mu_{l}$, shown with the solid black line. Panel (b) shows the gaseous and liquid densities, $\rho_{g}$ and $\rho_{l}$, with the ratio of the two on the right-hand $y$ axis. Panels (c) and (d) show the surface tension coefficient $\sigma$ and saturation pressure $P_{\text {sat. }}$. These plots are made from data compiled from previous studies [63-65].

In this study we have found the unique properties of superfluid and normal ${ }^{4} \mathrm{He}$ to offer several advantages for increasing our understanding of jet breakup. By varying its temperature within the small range between 1.2 and $5.2 \mathrm{~K}$ its fluid properties change dramatically passing through two transitions: the $\lambda$ point and the critical point. Below the $\lambda$ point, $T=2.17 \mathrm{~K}$, liquid ${ }^{4} \mathrm{He}$ is superfluid, possessing extremely low viscosity, which changes by two orders of magnitude [Fig. 3(a)]. As the temperature approaches the critical point, $T=5.19 \mathrm{~K}$, the saturated gas density $\rho_{g}$ approaches the liquid density $\rho_{l}$, thereby changing by three orders of magnitude and the surface tension coefficient $\sigma$ drops to zero at the critical point [Figs. 3(b)-3(d)]. Hence, by increasing the temperature, for fixed $d_{j}, \mathrm{Oh}_{l}$ and $\rho_{g} / \rho_{l}$ increase simultaneously in the range of $\mathrm{Oh}_{l} \sim O\left(10^{-5}-10^{-2}\right)$ and $\rho_{g} / \rho_{l} \sim$ $O\left(10^{-4}-1\right)$. Varying the jet exit velocity allows $\operatorname{Re}_{l}$ to change in the range of $\operatorname{Re}_{l} \sim O\left(10^{2}-10^{6}\right)$. This enables us to use one device to investigate a wide range of jet behaviors and study the regime transitions over the three-dimensional parameter space of $\mathrm{Re}_{l}, \mathrm{Oh}_{l}$, and $\rho_{g} / \rho_{l}$ discussed by Reitz and Bracco [27] and Torda [62].

\section{EXPERIMENTAL SETUP}

We perform the jet breakup experiments inside a cryostat with optical access that can achieve a minimum temperature of $1.2 \mathrm{~K}$. Figure 4 shows a diagram of the experimental setup. The cryostat cools three thermally isolated copper plates in stages. First, a Pfeiffer HiCube 300 turbomolecular pump pulls a vacuum inside the outer chamber to an absolute pressure of approximately $5 \times 10^{-7}$ torr minimizing convective and conductive heat transfer by the intervening gas. A Cryomech PT410 two-stage pulse-tube cryorefrigerator cools the first copper plate to $40 \mathrm{~K}$ and the second to $4 \mathrm{~K}$. Then evaporative cooling with ${ }^{4} \mathrm{He}$ cools the $1 \mathrm{~K}$ plate to a minimum temperature of $1.2 \mathrm{~K}$. Two finely polished, gold-plated, copper radiation shields connect to the bottom of the 40 and $4 \mathrm{~K}$ plates to reduce radiation heat transfer inside the chamber. We perform the experiments inside a cell that thermally sinks to the $1 \mathrm{~K}$ plate and has inner dimensions of approximately 4 by $4 \mathrm{~cm}^{2}$ in the horizontal directions and a height of $6 \mathrm{~cm}$. Calibrated thermometers mount to the 40, 4, 


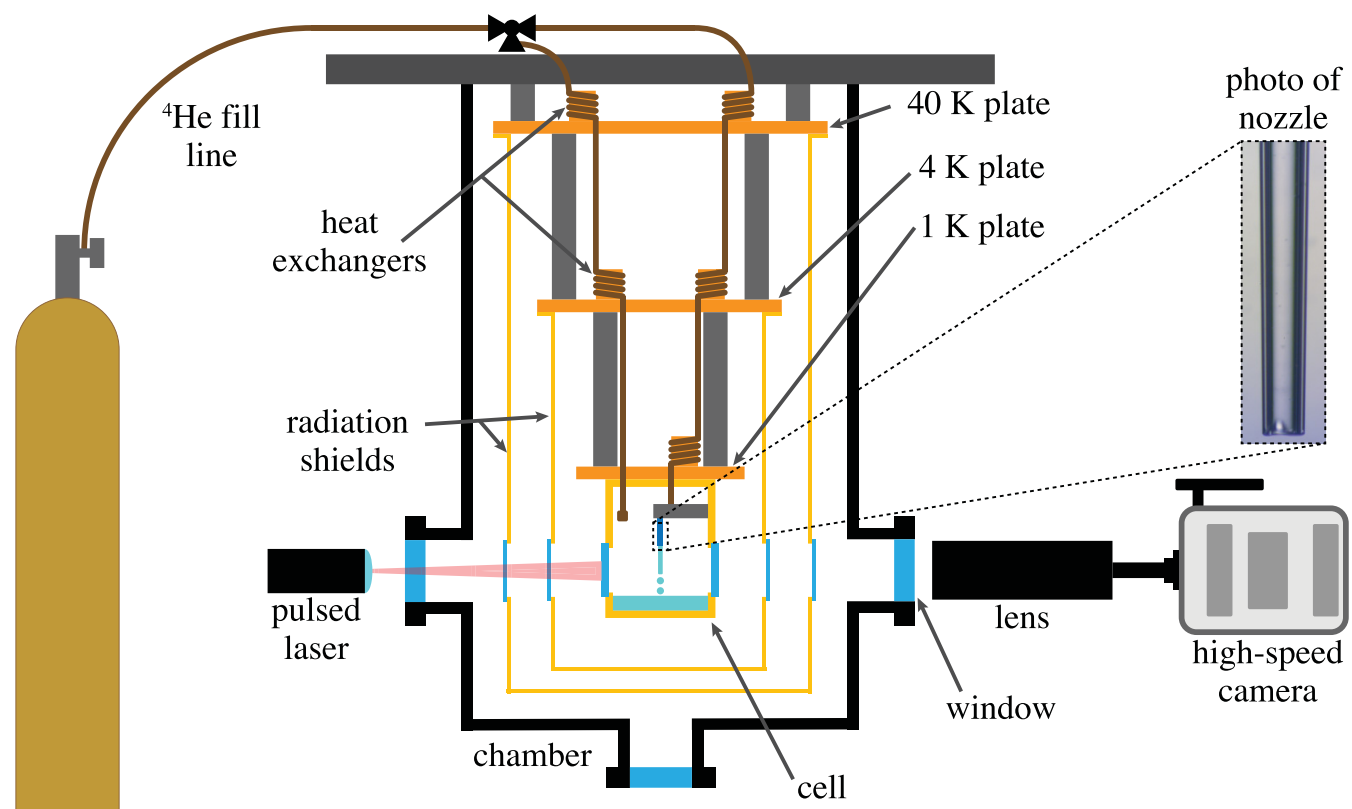

${ }^{4} \mathrm{He}$ pressure

cylinder

FIG. 4. A schematic of the basic experimental setup is shown. The cryostat consists of an outer chamber that holds a high vacuum. The cooling occurs in stages on the 40, 4, and $1 \mathrm{~K}$ plates, which indicate the nominal temperature values. Radiation shields connect to the bottom of the 40 and $4 \mathrm{~K}$ plates. The cell connects to the bottom of the $1 \mathrm{~K}$ plate to which it thermally sinks. The cell fills from a ${ }^{4} \mathrm{He}$ pressure cylinder through a line that cools on the 40 and $4 \mathrm{~K}$ plates through heat exchangers. The same pressure cylinder feeds the nozzle of the jet through a different line that cools on the 40,4 , and $1 \mathrm{~K}$ plates. The inset shows a photo of the tip of the glass nozzle. The breakup event is viewed with a high speed camera and long distance microscope lens with a pulsed laser for lighting.

and $1 \mathrm{~K}$ plates and an additional thermometer mounts to the inside of the cell wall. A Lakeshore 336 temperature controller monitors the temperature of each thermometer and provides PID control of the $1 \mathrm{~K}$ and cell temperatures in the range of $1.2-5.43 \mathrm{~K}$ via a small resistive heater mounted to the $1 \mathrm{~K}$ plate. Saturated conditions are maintained in the cell. Thus, the cell temperature determines the fluid properties of the liquid and gaseous helium, which are shown along the saturation curve as functions of the temperature in Figs. 3(a)-3(d) (data for fluid properties has been compiled from Refs. [63-65]).

Below the $\lambda$ transition $\left(T=2.17 \mathrm{~K}\right.$ ), liquid ${ }^{4} \mathrm{He}$ can begin to exhibit superfluid behavior. A two fluid model describes how the superfluid component coexists with the normal liquid helium. The normal fluid is dissipative and carries entropy while the superfluid does not. In some instances, such as a thermal counter flow, the normal and superfluid components can move separately from one another with little to no dissipation in the superfluid motion [66,67] (dissipation can occur when a mutual friction between the two components produces an apparent viscosity [68] or when the flow exceeds a critical velocity [69]). In other cases, the two components flow together with the normal component providing a small amount of viscous dissipation to the motion. Our experiments fall in the latter category. Therefore, in the calculation of our nondimensional parameters, we consider the combined liquid density, $\rho_{l}=\rho_{s}+\rho_{n}$, where $n$ and $s$ subscripts denote the normal and superfluid components, and the effective viscosity based on the normal fluid fraction, $\mu_{l}=\mu_{n} \rho_{n} / \rho_{l}$, as done 
by others [70]. Using this effective viscosity is a reasonable first approximation for the combined flow.

Before performing experiments we fill the cell with grade $5{ }^{4} \mathrm{He}(99.999 \%$ pure) from a pressure cylinder. The ${ }^{4} \mathrm{He}$ cools in heat exchangers on the 40 and $4 \mathrm{~K}$ plates and then passes through a filter with a pore size of $0.5 \mu \mathrm{m}$ that removes unwanted impurities. We maintain a ${ }^{4} \mathrm{He}$ pool at the bottom of the cell throughout the experiments to ensure that the cell stays at the saturated vapor pressure, which was verified with an absolute pressure gauge connected to the cell. This minimizes evaporation from the jet over the course of the experiments. The jet forms at the tip of a glass nozzle shown in the photograph in the inset of Fig. 4. The same pressure cylinder feeds the nozzle with ${ }^{4} \mathrm{He}$ through tubing that precools the helium on all three plates ensuring that the helium enters the cell at the same temperature as the cell and the $1 \mathrm{~K}$ plate. The nozzle is made by pulling a 1.5 -mm outer diameter, with 1.1-mm inner diameter, glass capillary tube with a Sutter P 1000 micropipette puller. With this method we formed a nozzle that has a straight section of inner diameter $1.1 \mathrm{~mm}$ followed by a rapid contraction and then a long tapered section whose diameter decreases gradually with a $2^{\circ}$ half cone angle to a finally inner diameter of $d_{j}=52 \mu \mathrm{m}$ and outer diameter of $72 \mu \mathrm{m}$. This final nozzle section has a length to diameter ratio $l / d_{j} \approx 60$ which should ensure that the helium exits the nozzle with a fully developed pipe flow. As the stream exits the nozzle we view the breakup with one of three high-speed cameras (Phantom V2511, Phantom V2640, or Specialised Imaging Kirana M5) at up to 5000000 frames per second. The camera looks through a Questar QM 100 long distance microscope lens which provides 1.2 to $5.0 \mu \mathrm{m} /$ pix resolution depending on the magnifying lenses and camera combination used. A pulsed diode laser (SILUX-640, Specialised Imaging) syncs with the camera providing $50 \mathrm{~ns}$ duration backlighting for each frame.

We control the jet exit velocity $U_{j}$ by adjusting the pressure drop across the nozzle using a pressure regulator on the pressure cylinder and a needle valve located on the nozzle feed line. Absolute pressure gauges measure the pressure in the nozzle feed line and in the cell. Prior to capturing data, the nozzle feed line pressure is allowed to stabilize producing a steady-state jet. Note that the continuous flow of helium into the cell increases the heat load on the $1 \mathrm{~K}$ plate and can raise the cell temperature altering other liquid and gas properties on the timescale of tens of seconds to minutes; however, each experimental run is on the timescale of milliseconds. To estimate $U_{j}$ we track features on the jet, such as the leading edge, bends and buckles, and other pinched-off locations. Four features of each jet are tracked with the mean value reported herein. At the highest velocities, generally in the second wind and atomization regimes, feature tracking does not result in accurate velocities due to the fine features associated with these breakup regimes. For these cases we estimate the velocity by fitting a curve to the pressure drop versus velocity plot for the measurable data and use the resulting least squares regression equation to calculate the jet velocity from the pressure drop. We estimate the uncertainty in the jet velocities from the standard deviation of the measured values and from using a regression curve for the calculated values. These uncertainties, as well as small uncertainties in the cell temperature are propagated to the appropriate nondimensional numbers using Taylor's method and Monte Carlo analysis [71] and are reported in the appropriate figures.

This setup allows us to vary a number of the important dimensional parameters for jet breakup. The important parameters include: nozzle inner diameter $d_{j}$, nozzle outer diameter $d_{\text {out }}$, nozzle length $L_{n}$, jet velocity $U_{j}$, surface tension coefficient $\sigma$, jet (liquid) density $\rho_{l}$, ambient (gas) density $\rho_{g}$, jet (liquid) viscosity $\mu_{l}$, and ambient (gas) viscosity $\mu_{g}$. These nine parameters have three dimensions (length, time, and mass) producing $9-3=6$ independent dimensionless groups. The six base groups could be given as the liquid Reynolds number $\operatorname{Re}_{l}=\rho_{l} d_{j} U_{j} / \mu_{l}$, the liquid Ohnesorge number $\mathrm{Oh}_{l}=\mu_{l} /\left(\rho_{l} \sigma d_{j}\right)^{1 / 2}$, the density ratio $\rho_{g} / \rho_{l}$, the viscosity ratio $\mu_{g} / \mu_{l}$, the nozzle diameter ratio $d_{j} / d_{\text {out }}$, and the nozzle aspect ratio $L_{n} / d_{j}$. Of these $\mathrm{Re}_{l}, \mathrm{Oh}_{l}$, and $\rho_{g} / \rho_{l}$ can combine to produce the liquid Weber number $\mathrm{We}_{l}=\mathrm{Oh}_{l}^{2} \mathrm{Re}_{l}^{2}$ and gas Weber number $\mathrm{We}_{g}=$ $\mathrm{Oh}_{l}^{2} \operatorname{Re}_{l}^{2}\left(\rho_{g} / \rho_{l}\right)$, which all help to describe the breakup regimes. Our viscosity ratio $\mu_{g} / \mu_{l}$ is $O(0.1)$ [Fig. 3(a)] and is of little effect due to the extremely low values of both phases. The 
nozzle diameter ratio $d_{j} / d_{\text {out }}$, held constant at 0.7 , becomes important when the liquid pins to the inner or outer diameters [29]. The nozzle aspect ratio $L_{n} / d_{j}$, also held constant, affects the velocity profile at the exit which can alter jet surface roughness and breakup behaviors [33]. Of the numerous dimensionless groups the most applicable for this study are $\operatorname{Re}_{l}, \mathrm{Oh}_{l}$, and $\rho_{g} / \rho_{l}$, with their combinations $\mathrm{We}_{l}$ and $\mathrm{We}_{g}$, which we will employ throughout this study. Hence, as we investigate the breakup regimes we focus on the three-dimensional parameter space defined by varying $\operatorname{Re}_{l}$, $\mathrm{Oh}_{l}$, and $\rho_{g} / \rho_{l}$.

\section{BREAKUP BEHAVIOR AND REGIMES}

A large range of jet velocities was examined for multiple cell temperatures. This produced an expansive range of $\mathrm{Re}_{l}, \mathrm{Oh}_{l}$, and $\rho_{g} / \rho_{l}$ over which we could examine the different breakup regimes. Figure 5 shows the progression of jet breakup behavior with increasing $\operatorname{Re}_{l}$ for three narrow $\mathrm{Oh}_{l}$ ranges at the (a) high, (b) medium, and (c) low ends of our parameter space. In the lowest $\mathrm{Oh}_{l} \mathrm{range}$ $\left(7.60 \times 10^{-5}-9.87 \times 10^{-5}\right)$, Fig. $5(\mathrm{c})$, jets break up in the Rayleigh regime at the lowest Reynolds numbers, $\operatorname{Re}_{l}=1.28 \times 10^{4}$ and $2.43 \times 10^{4}$, with droplets generally pinching-off one at a time near the bottom tip of the jet. Figure 6(a) and Supplemental Material video 1 [72] provide more detail of the first jet in Fig. 5(c) showing a time sequence that demonstrates the atypical lack of symmetry for a Rayleigh jet in this parameter space. This asymmetry is due to the extremely low viscosity of the superfluid which causes the jets to exit the nozzle with turbulent velocity fluctuations that distort the free surface and cause the jet to wobble back and forth. As $\operatorname{Re}_{l}$ increases we see first wind jets that have the defining characteristic of necking in multiple adjacent locations simultaneously leading to multiple pinch off events in rapid succession that do not always occur at the bottom tip of the jet as shown in Fig. 5(c) at $\operatorname{Re}_{l}=5.17 \times 10^{4}-5.03 \times 10^{5}$ and in the time sequence in Fig. 6(b). The surface roughness of these jets increases with $\mathrm{Re}_{l}$ as the turbulence from the pipe flow increases. Near the upper end of this first wind range some small droplets (known as entrainment droplets in Ref. [17]) pinch off from the sides of the jet which can be seen surrounding the core at $\operatorname{Re}_{l}=5.03 \times 10^{5}$. The quantity of the side droplets increases with $\operatorname{Re}_{l}$ and the core transitions to the sinuous breakup mode, which occurs in the range of $8.22 \times 10^{5}-1.5 \times 10^{6}$. These sinuous jets are distinguished by their fundamental asymmetry with the jet core forming twists and turns. At the highest $\operatorname{Re}_{l}$ shown $\left[\operatorname{Re}_{l}=1.50 \times 10^{6}\right.$ in Fig. 5(c), with a time sequence of the same jet in Fig. 6(c)], a significant portion of the jet liquid breaks off from the jet as side droplets, which reduces the diameter of the core. The jet core still breaks up in the sinuous mode, but breakup occurs more rapidly due to the diminished diameter forming smaller drops.

In the middle $\mathrm{Oh}_{l}$ range, shown in Fig. 5(b) $\left(\mathrm{Oh}_{l}=8.90 \times 10^{-4}-1.09 \times 10^{-3}\right)$, we see a similar regime progression to the lowest $\mathrm{Oh}_{l}$ range, but the transitions occur at different $\operatorname{Re}_{l}$. Some notable differences are that side droplets do not begin to form until the jet core breaks up in the sinuous mode and at the highest $\operatorname{Re}_{l}$ the quantity of side drops breaking off from the jet periphery has increased so much that larger drops no longer break off from the core, which is where we define the start of the second wind and atomization breakup regimes.

The highest $\mathrm{Oh}_{l}$ range $\left(\mathrm{Oh}_{l}=1.58 \times 10^{-2}-2.54 \times 10^{-2}\right)$, shown in Fig. 5(a), occurs at the highest temperatures, when the fluid in the cell is close to the critical point. Hence, in this study, high $\mathrm{Oh}_{l}$ also corresponds to high density ratio $\rho_{g} / \rho_{l}$ (close to 1) which also greatly affects the behavior of the jets, as discussed before in Sec. I. In this $\mathrm{Oh}_{l}$ range the Rayleigh jets are more axisymmetric due to the laminar pipe flow in the nozzle as indicated by the low $\operatorname{Re}_{l}$ [see Fig. 5(a), $\left.\operatorname{Re}_{l}=1.59 \times 10^{2}\right]$. At approximately the same $\operatorname{Re}_{l}$, but slightly higher $\mathrm{Oh}_{l}$ and $\rho_{g} / \rho_{l}$, the jets neck at multiple locations simultaneously and break up in the first wind mode $\left(\operatorname{Re}_{l}=1.56 \times 10^{2}\right)$. In this $\mathrm{Oh}_{l}$ range first wind only occurs for a narrow range of $\mathrm{Re}_{l}$ before transitioning to sinuous breakup. In these cases sinuous breakup $\left(\operatorname{Re}_{l}=2.15 \times 10^{2}-2.00 \times 10^{3}\right)$ appears somewhat different than at the lower $\mathrm{Oh}_{l}$ and $\rho_{g} / \rho_{l}$. The high gas density increases the buoyancy force on the jet and decelerates the droplets that have pinched off, grouping them together into a conical spray that begins to hide the jet core at the higher $\operatorname{Re}_{l}$ [Fig. $5(\mathrm{a}), \operatorname{Re}_{l}=5.15 \times 10^{2}-2.00 \times 10^{3}$ ]. As $\operatorname{Re}_{l}$ increases 


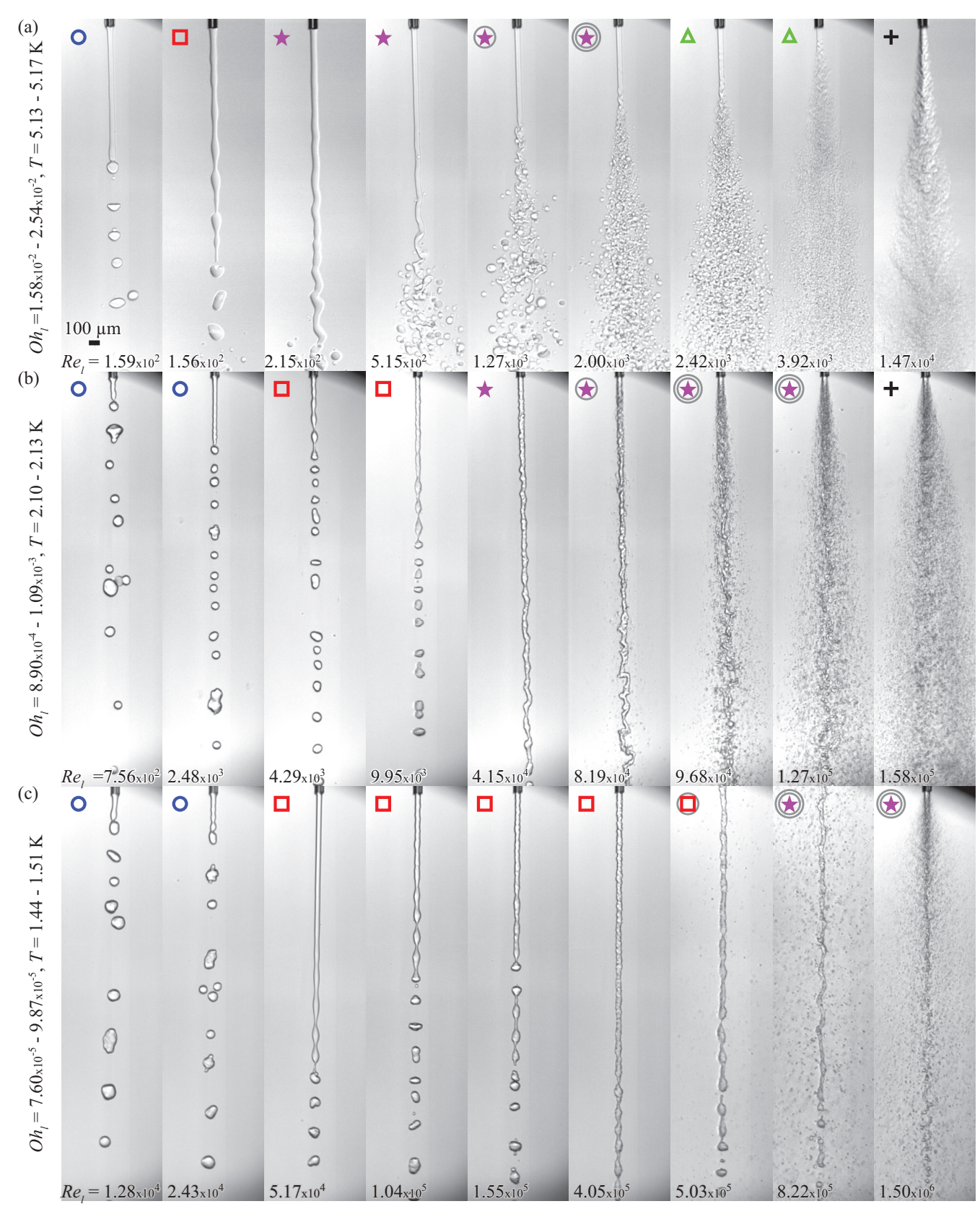

FIG. 5. Photos of the progression of jet breakup regimes are shown for three different $\mathrm{Oh}_{l}$ ranges as indicated to the left of each row ( $T$ ranges are also shown for each row). The $\operatorname{Re}_{l}$ for each case is shown at the bottom of each frame which generally increases from left to right. The symbols at the top left of each frame indicate the breakup regime classification and correspond to the symbols in the regime diagram in Fig. 7. Blue circles $\bigcirc$ are Rayleigh breakup, red squares $\square$ are first wind, magenta stars $\star$ are sinuous, green triangles $\triangle$ are second wind, and black pluses + are atomization. Gray circles $\bigcirc$ surrounding the symbol indicate the approximate quantity of side drops with one circle for some and two for a lot. The dark triangle in the top right of some frames is vignetting from the microscope lens. 

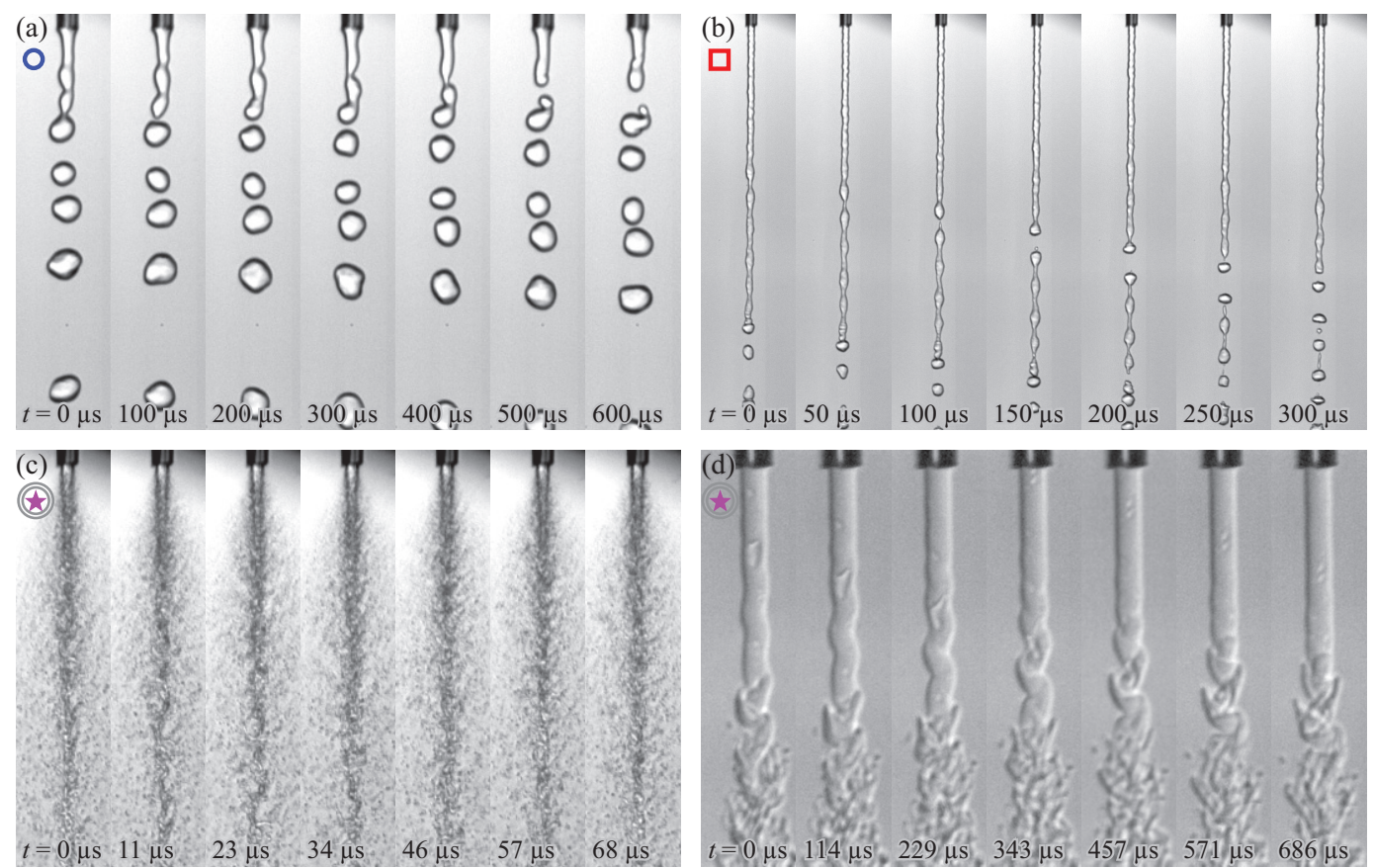

FIG. 6. Time sequences of four jets are shown. In panel (a) a superfluid jet exits the nozzle at $U_{j}=0.20 \mathrm{~m} / \mathrm{s}$ and $T=1.44 \mathrm{~K}$ which results in Rayleigh breakup at $\mathrm{Oh}_{l}=7.60 \times 10^{-5}$ and $\mathrm{Re}_{l}=1.28 \times 10^{4}$. In panel (b) a superfluid jet exits the nozzle at $U_{j}=2.52 \mathrm{~m} / \mathrm{s}$ and $T=1.45 \mathrm{~K}$ which results in first wind breakup at $\mathrm{Oh}_{l}=7.90 \times 10^{-5}$ and $\mathrm{Re}_{l}=1.55 \times 10^{5}$. In panel (c) a superfluid jet exits the nozzle at $U_{j}=26.35 \mathrm{~m} / \mathrm{s}$ and $T=1.47 \mathrm{~K}$ which results in sinuous breakup of the core with a lot of side droplets at $\mathrm{Oh}_{l}=8.51 \times 10^{-5}$ and $\operatorname{Re}_{l}=1.50 \times 10^{6}$. In panel (d) a normal fluid jet near the critical point exits the nozzle at $U_{j}=0.61 \mathrm{~m} / \mathrm{s}$ and $T=5.17 \mathrm{~K}$ which results in a sinuous breakup near the transition to second wind at $\mathrm{Oh}_{l}=2.54 \times 10^{-2}$ and $\operatorname{Re}_{l}=1.18 \times 10^{3}$. Some bubble can be seen inside the jet which are more common near the critical point. For scale the nozzle's outer diameter at the tip is $72 \mu \mathrm{m}$ in each image. See Supplemental Material videos 1-4 that correspond to panels (a)-(d), respectively [72].

the sinuous windings and wrinkles become tighter, making sharper turns, and the jet breaks up more quickly into increasingly smaller droplets $\left(\operatorname{Re}_{l}=2.00 \times 10^{3}\right)$. Figure $6(\mathrm{~d})$ and Supplemental Material video 4 [72] show a zoomed in time sequence of a jet with $\mathrm{Oh}_{l}=2.54 \times 10^{-2}$ near the transition to second wind, demonstrating how the sinuous buckles smash together forming a jumbled mess of liquid ligaments that eventually break up into droplets. Breakup gradually transitions to the second wind regime as the windings disappear $\left(\operatorname{Re}_{l}=2.42 \times 10^{3}-3.92 \times 10^{3}\right)$. Sticking with a similar definition to Reitz's [6], we define the breakup as atomization when the jet breaks up within one nozzle diameter of the exit, which we see at the highest $\operatorname{Re}_{l}$. A summary of the defining characteristics for each regime is given in Table I.

In the low $\mathrm{Oh}_{l}$ range of this study, the interface of the liquid is expected to overturn close to the pinch-off event [30,73]. Close examination of Figs. 5 and 6(a) and 6(b) and the Supplemental Material videos 1 and 2 [72] provides no clear evidence of the overturning of the interface. Low spatial resolution relative to the size of the jet coupled with imaging perpendicular to the jet could be contributing factors in occluding the overturning event, if present. Additionally, after pinch off, the drops exhibit a multitude of interesting shapes owing to the low viscosity and thus low dissipation of the capillary waves on the surface of the drop. Drops exhibiting two-, three-, and even fivelobed shapes occur. Further study with larger drops or higher magnification is warranted to more fully understand the overturning of the interface and the droplet oscillation dynamics after pinch off. 
TABLE I. A summary of the regime names used in this study, the symbol, the defining visual characteristics, and the quantitative criteria for transition to the next regime are shown.

\begin{tabular}{llll}
\hline \hline Regime & Symbol & \multicolumn{1}{c}{ Defining visual characteristic } & Transition \\
\hline Rayleigh & $\wp$ & Single droplets pinch off from the leading end & $\mathrm{We}_{l}=8$ \\
First wind & $\square$ & Necking occurs in multiple adjacent locations simultaneously & $\mathrm{We}_{g}=8$ \\
Sinuous & $\star$ & Asymmetric twisting or corkscrew shape & $\mathrm{We}_{g}=120$ \\
Second wind & $\triangle$ & Small droplets break off from the periphery consuming the entire core & \\
Atomization & + & Second wind breakup that begins within one jet diameter of the nozzle & \\
\hline \hline
\end{tabular}

\section{TRANSITIONS CRITERIA BETWEEN BREAKUP REGIMES}

In Fig. 7(a) we plot over 300 data points on the traditional $\mathrm{Re}_{l}-\mathrm{Oh}_{l}$ regime diagram with the breakup modes indicated by different symbols and show, once again $[13,17]$, that the different breakup modes group together into specific locations. Remember that the temperature and pressure inside the cell were kept on the saturation curve, which causes $\rho_{g} / \rho_{l}$ to have a one-to-one correspondence with $\mathrm{Oh}_{l}$ as shown in Fig. 7(b). Hence, as $\mathrm{Oh}_{l}$ increases, $\rho_{g} / \rho_{l}$ increases as well and we obtain a three-dimensional surface of information on the breakup regimes. As will be shown, the continuous variation of the density ratio $\rho_{g} / \rho_{l}$ enables us to distinguish between effects of the gas and liquid, but the pairing of $\mathrm{Oh}_{l}$ and $\rho_{g} / \rho_{l}$ makes it more difficult to determine which of the two variables causes the change in jet behavior. We attempt to overcome this by comparing with

(a)

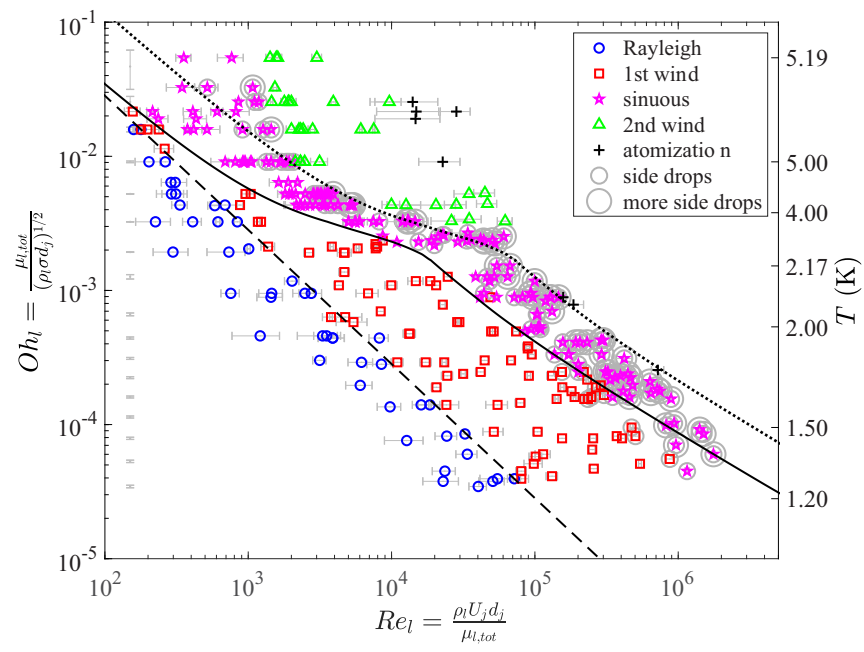

(b)

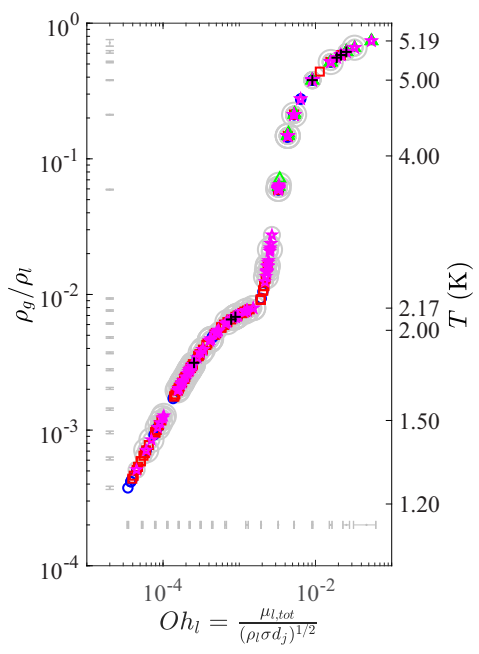

FIG. 7. (a) The different modes of jet breakup can be divided on a $\mathrm{Re}_{l}-\mathrm{Oh}_{l}$ regime diagram similar to the data of Ohnesorge [13] and Saito et al. [17]. As $\mathrm{Oh}_{l}$ and $\rho_{g} / \rho_{l}$ are both functions of $T$ for constant $d_{j}$ the relationship between the two is shown in panel (b) showing the three-dimensionality of the regime diagram. The right-hand axes of panels (a) and (b) show the corresponding $T$ to the $\mathrm{Oh}_{l}$ and $\rho_{g} / \rho_{l}$ on the left-hand axes. The small circle, square, star, triangle, and plus symbols indicate the mode of breakup of the core of the jet and the medium and large gray circles surrounding these symbols indicate if droplets pinch off from the sides of the jet, with one gray circle indicating some droplets and two indicating a lot as shown in Fig. 5. Gray circles have been omitted from the second wind and atomization regimes to reduce clutter and because side droplets are the cause of the breakup of the core of the jet in these cases. The dashed line "--" plots $\mathrm{We}_{l}=8$, the solid line "-." plots $\mathrm{We}_{g}=8$, and the dotted line “..." plots $\mathrm{We}_{g}=120$. 

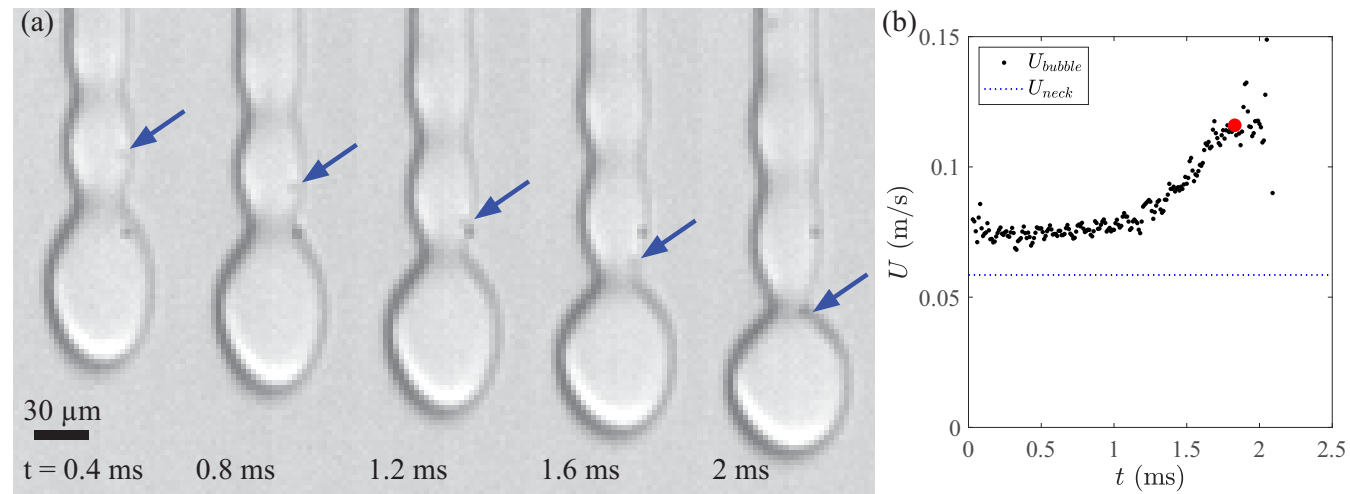

FIG. 8. (a) A small bubble inside a jet with $\mathrm{We}_{l}=8$ moves downward relative to the jet interface as indicated by the blue arrows. The velocities of the bubble and neck are shown over time in panel (b). The large red dot in panel (b) indicates the time that the bubble passes through the narrowest portion of the neck. The time $t$ at the bottom of the frames in panel (a) correspond to the time in panel (b). The small stationary spot in panel (a) is a dust particle on the camera lens.

previous studies. Using this three-dimensional plot, we discuss the transitions between the regimes and compare with the literature.

Rayleigh jets transition to first wind when the flow over the undulating surface of the jet accentuates the effect of the surface tension pressure with a Bernoulli pressure. Assuming that both the liquid and surrounding gas flow over the interface we expect the liquid to exert a much larger Bernoulli pressure $\left(\rho U^{2} / 2\right)$ due to its significantly higher density [Fig. 3(b)] Hence, first-wind jets should start to appear when the characteristic Bernoulli pressure of the liquid is approximately equal to the characteristic capillary pressure. Equating the two, using two curvatures (a maximum pressure) for the capillary pressure, we obtain

$$
\frac{\rho_{l} U_{j}^{2}}{2} \approx \frac{4 \sigma}{d_{j}},
$$

which after rearranging yields $\mathrm{We}_{l} \approx 8$. We note that a similar expression can be obtained by comparing the Rayleigh pinch off timescale $\left(\rho_{l} d_{j}^{3} / \sigma\right)^{1 / 2}[3,29]$ to the flow timescale $d_{j} / U_{j}$ which leads to $\mathrm{We}_{l} \sim 1$. To validate the assumption that the liquid in the jet moves relative to the interface, we track a small bubble inside a jet with $\mathrm{We}_{l}=8$ in Fig. 8(a) and see that it passes from a bulging to a necking location. In Fig. 8(b) we see that its velocity increases from approximately 0.07 to $0.12 \mathrm{~m} / \mathrm{s}$ in the converging flow. This qualitatively says that during the initial growth of the perturbation of the interface, long before pinch off, the pressure in the neck is lower than in the bulge and the Bernoulli pressure in the liquid draws the interface inward, toward the jet center, assisting the growth of the perturbation. Using the velocity of the bubble as it passes through the neck [red dot in Fig. 8(b)] and the two radii of curvature of the neck at that time, we can calculate the ratio of the Bernoulli pressure to the interfacial pressure and obtain $\left(\rho_{l} U_{\text {bubble }}^{2} / 2\right) /\left[\sigma\left(1 / R_{1}+1 / R_{2}\right)\right]=6$. This indicates that in the early stages of the pinch off the Bernoulli and interfacial pressures are of the same order in necking locations when $\mathrm{We}_{l} \approx 8$ and that we can expect the flow of the liquid to assist surface tension in the breakup of jets at $\mathrm{We}_{l} \gtrsim 8$. As the radius of the neck goes to zero, the capillary pressure in the neck rises above the pressure in the bulge expelling liquid in the region and allowing the pinch off to occur [74].

We plot $\mathrm{We}_{l}=8\left(\mathrm{Oh}_{l}=\sqrt{8} \mathrm{Re}_{l}^{-1}\right)$ in Fig. 7 (a) with the dashed line and see that it divides the Rayleigh and first wind regimes quite well. Seeing that an increase in the liquid flow demarcates this regime transition, it seems that the regime name of "first wind" may be a misnomer, as "wind" implies an effect of the gaseous phase. Perhaps naming the regime "first flow" would be a more 
accurate description. This transition criteria differs from the work of Ranz [15] who theorized that $\mathrm{We}_{l}=8$ should delineate the transition between dripping and Rayleigh jets and the reviews of Reitz et al. $[6,18,27,28]$ who predicted this transition with a gas Weber number, $\mathrm{We}_{g}$ (see Fig. 2). Note that for a constant density ratio both the liquid and gas Weber number would appear as straight lines on a $\log -\log \mathrm{Oh}_{l}$ versus $\mathrm{Re}_{l}$ plot obscuring which parameter is responsible for the breakup transition.

We now look at the transition from first wind to sinuous breakup. Haenlein [5] states that aerodynamic forces, from the gaseous phase, act against surface tension to form sinuous jets. This implies that sinuous breakup occurs when the Bernoulli pressure from the gas exceeds the interfacial pressure. Hence, as above, we equate the two, replacing $\rho_{l}$ with $\rho_{g}$ in Eq. (1), and after rearranging find that sinuous breakup should occur when $\mathrm{We}_{g} \gtrsim 8$. We plot $\mathrm{We}_{g}=8$ $\left(\mathrm{Oh}_{l}=\sqrt{8}\left(\rho_{g} / \rho_{l}\right)^{-1 / 2} \mathrm{Re}_{l}^{-1}\right)$ in Fig. 7(a) with a solid black line and find that it effectively divides the first wind and sinuous breakup regimes. Notice that as $\rho_{g} / \rho_{l} \longrightarrow 1$, at high $\mathrm{Oh}_{l}$ [Fig. 7(b)], $\mathrm{We}_{g} / \mathrm{We}_{l} \longrightarrow 1$ and the first wind breakup regime disappears [Fig. 7(a)]. This agrees well with the findings of Saito et al. [17] who investigated the breakup of a liquid jet in an immiscible liquid pool, with a density ratio close to 1 . They did not report the occurrence of first wind jets, which should only occur for a very narrow range of $\mathrm{Re}_{l}$ at their high density ratio. Rather they state that Rayleigh jets transition to sinuous when $\mathrm{Oh}_{l}=2.8 \mathrm{Re}_{l}^{-1}$ which rearranges to $\mathrm{We}_{l} \approx \mathrm{We}_{g} \approx 8$, for $\rho_{g} / \rho_{l} \approx 1$.

Entov and Yarin [7] theoretically showed that the growth of the bending (asymmetric) perturbations dominates the growth of the axisymmetric ones when two conditions are met. First, surface tension must be insignificant as indicated by $\mathrm{We}_{g} \gg 1$. Second, the jet liquid must be sufficiently viscous such that $4 \mu_{l}^{2}\left(\rho_{l} d_{j}^{2} \rho_{g} U_{j}^{2}\right)^{-1} \gg 1$. Although our sinuous jets occur at high $\mathrm{We}_{g}$ they are extremely inviscid with $4 \mu_{l}^{2}\left(\rho_{l} d_{j}^{2} \rho_{g} U_{j}^{2}\right)^{-1} \ll 1$. Hence, for our sinuous jets the axisymmetric perturbations grow along with the bending perturbations and the jets bend and bulge simultaneously [e.g., Fig. 5 (b), $\operatorname{Re}_{l}=4.15 \times 10^{4}$ ]. For comparison to a sinuous jet in a more viscous fluid see Haenlein's [5] sinuous jets with castor oil for which no bulging accompanies the bending.

The transition from sinuous to the second wind and atomization regimes is empirically found to occur at $\mathrm{We}_{g} \approx 120$ where the Bernoulli pressure is approximately 15 times the interfacial pressure. This once again agrees reasonably well with the findings of Saito et al. [17] in the high $\rho_{g} / \rho_{l}$ region of our regime diagram. Saito et al. report this transition as $\mathrm{Oh}_{l}=22 \mathrm{Re}_{l}^{-1}$ (not shown on our plot), which at high $\rho_{g} / \rho_{l}$ [high $\mathrm{Oh}_{l}$ in Fig. 7(a)], falls a little to the right of the $\mathrm{We}_{g}=120\left[\mathrm{Oh}_{l}=\right.$ $\left.\sqrt{120}\left(\rho_{g} / \rho_{l}\right)^{-1 / 2} \operatorname{Re}_{l}^{-1}\right]$ line we plot in Fig. 7(a). Due to the limitation of maintaining a constant cell temperature while imposing a high heat load at large injection flow rates, in this work we have fewer jets breaking up in the atomization mode and hence do not find a quantitative transition from second wind to atomization. This topic has been discussed in a previous work [6].

\section{JET BREAKUP LENGTH}

We now shift our focus to the breakup length $L_{b}$, measured from the nozzle to breakup position of the jet core, and examine how it changes for the various parameters and regime transitions. A common view on the breakup length of jets is discussed in the review by Lin and Reitz [18]. They state that at the lowest velocities the breakup length increases linearly with jet velocity $\left(L_{b}=U_{j} t_{b}\right.$, where $t_{b}$ is the breakup time) until inertial forces increase the rate of breakup, decrease $t_{b}$, thereby creating a local maximum. They define this local maximum as the transition from Rayleigh to first wind. The breakup length then decreases toward zero as jets approach the atomization regime with even larger velocities. Other studies [5,32,75] complicate the issue showing additional effects of the velocity profile and turbulence, which we now discuss with the data from our unique parameter space. Figure 9 shows a plot of the normalized jet breakup length, $L_{b} / d_{j}$, versus $\mathrm{We}_{g}$ for three ranges of $\mathrm{Oh}_{l}$ corresponding with the rows in Fig. 5. The reported values are an average breakup length from 10 frames with the accompanying error bars showing the uncertainty due to natural variation and droplets obscuring the view of the jet core. In some cases, $L_{b}$ was longer than the frame so we 


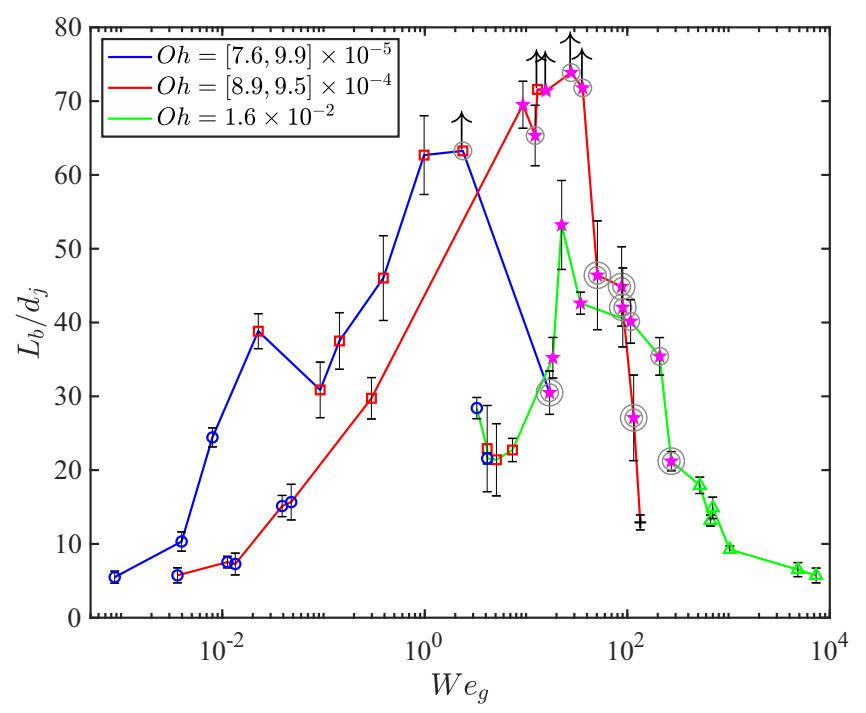

FIG. 9. The breakup length of the jet core $L_{b}$ normalized by the jet diameter $d_{j}$ is plotted against $\mathrm{We}_{g}$ for three different ranges of $\mathrm{Oh}_{l}$, which correspond to the three rows from Fig. 5. The green line corresponds with row (a), the red line with row (b), and the blue line with row (c). The symbols represent the breakup regime for each measurement as indicated in Fig. 7(a). The upward arrows associated with some points mark when the jet breakup length is longer than the image frame.

plot the frame length with an upward arrow to denote that the breakup occurs at some length larger than the marker.

Looking at the breakup length curve for the three $\mathrm{Oh}_{l}$ ranges we see that the curve for the lowest $\mathrm{Oh}_{l}$ range exhibits two local maxima (Fig. 9, blue line). The first corresponds with the transition to first wind as discussed by Lin and Reitz [18]. Shortly after this regime transition, the jet turbulence increases as seen by the ruffled appearance of the jet in Fig. 5 (c) for $\operatorname{Re}_{l} \geqslant 1.55 \times 10^{5}$. The increased turbulence causes the jet length to increase again toward the second local maximum, as discussed by Grant and Middleman [32], which also occurs in the first wind regime. At sufficiently high velocities, the jet length decreases; however, since we are unable to reach the second wind or atomization regimes for this $\mathrm{Oh}_{l}$ range, we do not observe the breakup length go to zero. For the middle $\mathrm{Oh}_{l}$ range (Fig. 9, red line), we only observe a single peak that occurs in the sinuous regime. It is possible that finer control over the velocity would elucidate a second maximum at the first wind transition, but such precise control over the velocity proved challenging with the current setup and such data evaded us. Additionally, the transition to turbulence occurs near the start of the first wind regime, which leaves little parameter space for a decrease in jet length and possibly masks any apparent peak at the transition to first wind. In this middle $\mathrm{Oh}_{l}$ range, we see the longest jets, and an abrupt decrease toward zero as jets transition to atomization. The highest $\mathrm{Oh}_{l}$ range (Fig. 9, green line) behaves similar to the middle range in that we only observe a single prominent peak that occurs in the sinuous regime. For increasing velocity, the transition to second wind or atomization occurs more smoothly than for the lower $\mathrm{Oh}_{l}$ cases, which may in part be attributed to the continued laminar flow of the jets into the second wind regime as seen with the smooth jet surfaces in Fig. 5(a).

Overall, the effect of increasing $\mathrm{Oh}_{l}$ and $\rho_{g} / \rho_{l}$ is a shifting of the breakup length curve and the maximum length to higher $\mathrm{We}_{g}$. The prominence of the first peak that is expected near the Rayleigh to first wind transition also diminishes to the point of not being visible at the higher $\mathrm{Oh}_{l}$ values. 


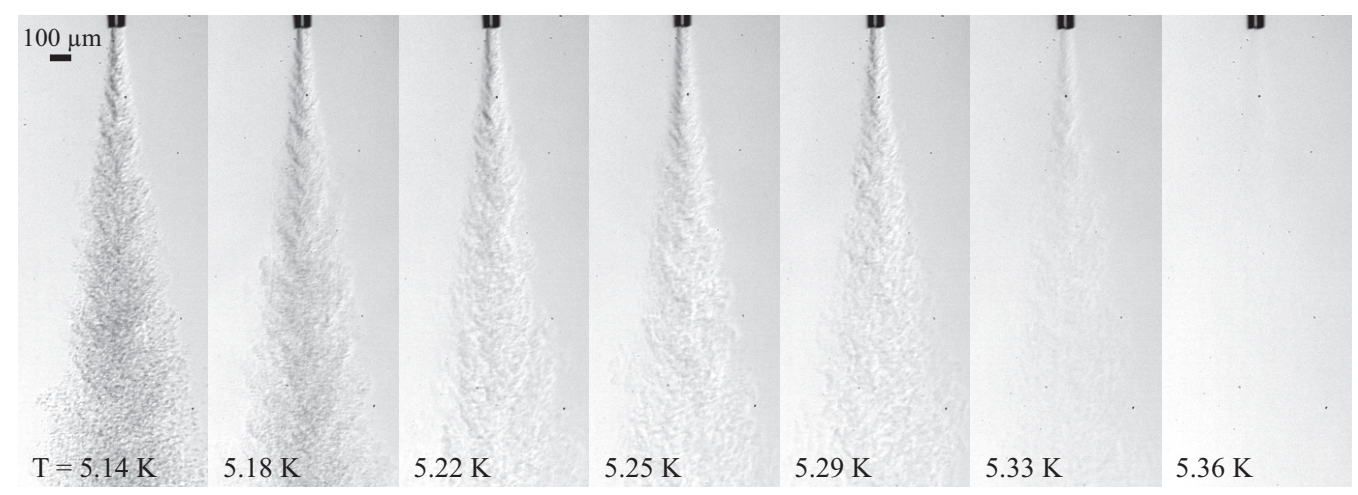

FIG. 10. Photos from a single event of a jet in the atomization regime as the cell temperature $T$ increases through the critical point, $T=5.19 \mathrm{~K}$. The cell temperature is shown at the bottom of each frame.

\section{THE CRITICAL POINT}

We now briefly investigate the breakup of jets at the critical point, $T=5.19 \mathrm{~K}$, where surface tension goes to zero $(\sigma \rightarrow 0)$ and the densities of the two phases converge $\left(\rho_{g} / \rho_{l} \rightarrow 1\right)$, followed by the disappearance of the liquid phase. To accomplish this we form an atomizing jet at a cell temperature of $T=5.14 \mathrm{~K}$ which increases gradually to $T=5.36 \mathrm{~K}$ over the course of about $2 \mathrm{~min}$, shown in Fig. 10. At $T=5.14 \mathrm{~K}$ we see distinct droplets in the atomizing spray, evidenced by the grainy appearance. The graininess decreases $(T=5.18 \mathrm{~K})$ as the temperature approaches the critical point. Once the cell has passed through the critical point a gaseous density distribution replaces the individual droplets as evidenced by the more blurred appearance at $T=5.22 \mathrm{~K}$. Above the critical point we see the turbulent structures of the gaseous jet appear qualitatively similar to the atomized spray of small droplets below the critical point but smooth gradients replace the distinct interfaces. The jet gradually disappears from our view as the temperature continues to increase.

\section{CONCLUSIONS}

In this study we have examined the breakup of liquid ${ }^{4} \mathrm{He}$ jets in a saturated atmosphere of its own vapor for a temperature range of 1.2 to $5.2 \mathrm{~K}$. This unique fluid has a large variation of several fluid properties over this small temperature range which enables the investigation of jet breakup over a wide range of key nondimensional parameters. The extremely low viscosity of liquid ${ }^{4} \mathrm{He}$ allows investigation in the limit of low $\mathrm{Oh}_{l}$ in the range of $O\left(10^{-5}-10^{-2}\right)$, while using a very small nozzle. As the temperature increases toward the critical point the gas-liquid density ratio $\rho_{g} / \rho_{l}$ increases from $O\left(10^{-4}\right)$ to $O(1)$. In addition to a large range of obtainable fluid properties our large range of jet velocities produced $\mathrm{Re}_{l} \sim O\left(10^{2}-10^{6}\right), \mathrm{We}_{l} \sim O\left(10^{-1}-10^{5}\right)$, and $\mathrm{We}_{g} \sim O\left(10^{-4}-10^{5}\right)$.

The nature of breakup varies over this large parameter space and we have shown that five distinct behaviors or regimes exist for the breakup of the jet core. Previous works have named these regimes Rayleigh, first wind, sinuous, second wind, and atomization, listed in the order of increasing jet velocity. We have qualitatively discussed the gradual evolution of the breakup behavior within regimes and the quantitative transitions between them. The transition from Rayleigh to first wind occurs as the jet liquid flows over the undulating interface and exerts a pressure approximately equal to the interfacial pressure, at $\mathrm{We}_{l} \approx 8$. Similarly, when the flow of the gas exerts a pressure approximately equal the interfacial pressure, at $\mathrm{We}_{g} \approx 8$, bending perturbations grow alongside the axisymetric ones and first wind breakup transitions to sinuous breakup. At higher velocities small droplets pinch off from the sides of first wind and sinuous jets. The rate of these small pinch off events increases until $\mathrm{We}_{g}=120$ when they consume the jet core and the entire jet breaks up into a spray of small droplets in the second wind and atomization regimes. 
We can expect that investigating other parameter spaces would reveal the existence of additional regimes of breakup behavior, likely omit some of the regimes we discuss, and alter transitions between them. For example, higher viscosity fluids suppress the growth of axisymmetric perturbations, but permit the growth of asymmetric ones [7]. This would alter the occurrence of Rayleigh and first wind jets and the appearance of the sinuous jets as shown in this study. Higher ambient viscosity in which $\mu_{g} / \mu_{l} \gtrsim 1$ or density ratios $\rho_{g} / \rho_{l}>1$ (larger ambient density) would greatly alter the drag on the interface and buoyancy forces, thereby likely altering breakup behaviors.

In this study we have also investigated the breakup length and breakup at the critical point. We have found that the maximum jet length shifts to higher $\mathrm{We}_{g}$ as the $\mathrm{Oh}_{l}$ or $\rho_{g} / \rho_{l}$ increases. Additionally, the prominence of the local maximum that is expected near the Rayleigh to first wind transition, may not persist at all $\rho_{g} / \rho_{l}$ values. We have seen that an atomizing liquid spray transitions to a turbulent gaseous jet at the critical point.

The findings in this paper improve our understanding of the breakup of liquid jets and connect the breakup regimes previously observed separately in gaseous [13] and liquid [17] atmospheres. This work may apply to the development of combustion processes in which fuel injection occurs near a liquid-vapor critical point [76], to inkjet printing, or to squirt gun fights with friends.

\section{ACKNOWLEDGMENTS}

This work was supported by King Abdullah University of Science and Technology (KAUST) under Grant No. URF/1/2621-01-01.

[1] J. Plateau, LI. Experimental and theoretical researches on the figures of equilibrium of a liquid mass withdrawn from the action of gravity—-third series, London Edinburgh Dublin Philos. Mag. J. Sci. 14, 431 (1857).

[2] L. Rayleigh, On the instability of jets, Proc. London Math. Soc. s1-10, 4 (1878).

[3] L. Rayleigh, On the capillary phenomena of jets, Proc. R. Soc. London 29, 71 (1879).

[4] L. Rayleigh, On the instability of cylindrical fluid surfaces, London Edinburgh Dublin Philos. Mag. J. Sci. 34, 177 (1892).

[5] A. Haenlein, Disintegration of a liquid jet, Tech. Rep. NACA-TM-659 (Forschung auf dem Gebiet des Ingenieurwesens A, Washington, DC, 1932).

[6] R. D. Reitz, Atomization and other breakup regimes of a liquid jet, Ph.D. thesis, Princeton University, 1978.

[7] V. M. Entov and A. L. Yarin, The dynamics of thin liquid jets in air, J. Fluid Mech. 140, 91 (1984).

[8] A. L. Yarin, Free Liquid Jets and Films: Hydrodynamics and Rheology (Longman, Wiley \& Sons; Harlow/New York, 1993).

[9] A. L. Yarin, Bending and buckling instabilities of free liquid jets: Experiments and general quasi-onedimensional mode, in Handbook of Atomization and Sprays: Theory and Applications, edited by N. Ashgriz (Springer Science \& Business Media, Berlin, 2011).

[10] C. Weber, Zum zerfall eines flüssigkeitsstrahles, J. Appl. Math. Mech./Zeitschrift für Angewandte Mathematik und Mechanik 11, 136 (1931).

[11] P. Debye and J. Daen, Stability considerations on nonviscous jets exhibiting surface or body tension, Phys. Fluids 2, 416 (1959).

[12] V. M. Entov and A. L. Yarin, Dynamical equations for a liquid jet, Fluid Dyn. 15, 644 (1980).

[13] W. V. Ohnesorge, Die bildung von tropfen an düsen und die auflösung flüssiger strahlen, J. Appl. Math. Mech./Zeitschrift für Angewandte Mathematik und Mechanik 16, 355 (1936).

[14] A. C. Merrington and E. G. Richardson, The break-up of liquid jets, Proc. Phys. Soc. 59, 1 (1947).

[15] W. E. Ranz, On sprays and spraying, Bulletin (Department of Engineering Research, Pennsylvania State University, 1956). 
[16] N. I. Kolev, Liquid and gas jet disintegration, in Multiphase Flow Dynamics 2 (Springer, Berlin/Heidelberg, 2005), pp. 273-296.

[17] S. Saito, Y. Abe, and K. Koyama, Flow transition criteria of a liquid jet into a liquid pool, Nucl. Eng. Des. 315, 128 (2017).

[18] S. P. Lin and R. D. Reitz, Drop and spray formation from a liquid jet, Annu. Rev. Fluid Mech. 30, 85 (1998).

[19] S. Saito and Y. Abe, and K. Koyama, Visualization and measurement of jet breakup behavior using LIF, Nippon Kikai Gakkai Ronbunshu 81, 832 (2015).

[20] S. Chandrasekhar, Hydrodynamic and Hydromagnetic Stability (Oxford University Press, Oxford, UK, 1961).

[21] J. Buckmaster, The buckling of thin viscous jets, J. Fluid Mech. 61, 449 (1973).

[22] A. A. Castrejòn-Pita, J. R. Castrejòn-Pita, and I. M. Hutchings, Breakup of Liquid Filaments, Phys. Rev. Lett. 108, 074506 (2012).

[23] D. W. Lee and R. C. Spencer, Photomicrographic studies of fuel sprays, Tech. Rep. 454 ( National advisory committee for aeronautics, 1933).

[24] C. C. Miesse, Correlation of experimental data on the disintegration of liquid jets, Ind. Eng. Chem. 47, 1690 (1955).

[25] A. Haenlein, Über den zerfall eines flüssigkeitsstrahles, Forschung auf dem Gebiet des Ingenieurwesens A 2, 139 (1931).

[26] G. H. McKinley and M. Renardy, Wolfgang von ohnesorge, Phys. Fluids 23, 127101 (2011).

[27] R. D. Reitz and F. V. Bracco, Mechanisms of breakup of round liquid jets, in The Encyclopedia of Fluid Mechanics (Gulf Publishing, Houston, TX, 1986).

[28] N. Chigier and R. D. Reitz, Regimes of jet breakup and breakup mechanisms (physical aspects), in Recent Advances in Spray Combustion: Spray Atomization and Drop Burning Phenomena, edited by K. K. Kuo (American Institute of Aeronautics and Astronautics, Reston, VA, 1995), Vol. 1, pp. 109-135.

[29] C. Clanet and J. C. Lasheras, Transition from dripping to jetting, J. Fluid Mech. 383, 307 (1999).

[30] B. Ambravaneswaran, H. J. Subramani, S. D. Phillips, and O. A. Basaran, Dripping-Jetting Transitions in a Dripping Faucet, Phys. Rev. Lett. 93, 034501 (2004).

[31] A. M. Sterling and C. A. Sleicher, The instability of capillary jets, J. Fluid Mech. 68, 477 (1975).

[32] R. P. Grant and S. Middleman, Newtonian jet stability, AIChE J. 12, 669 (1966).

[33] M. J. McCarthy and N. A. Molloy, Review of stability of liquid jets and the influence of nozzle design, Chem. Eng. J. 7, 1 (1974).

[34] P. K. Notz and O. A. Basaran, Dynamics and breakup of a contracting liquid filament, J. Fluid Mech. 512, 223 (2004).

[35] F. Wang, F. P. Contò, N. Naz, J. R. Castrejón-Pita, A. A. Castrejón-Pita, C. G. Bailey, W. Wang, J. J. Feng, and Y. Sui, A fate-alternating transitional regime in contracting liquid filaments, J. Fluid Mech. 860, 640 (2019).

[36] C. R. Anthony, P. M. Kamat, M. T. Harris, and O. A. Basaran, Dynamics of contracting filaments, Phys. Rev. Fluids 4, 093601 (2019).

[37] R. M. S. M. Schulkes, The contraction of liquid filaments, J. Fluid Mech. 309, 277 (1996).

[38] E. K. Longmire, T. L. Norman, and D. L. Gefroh, Dynamics of pinch-off in liquid/liquid jets with surface tension, Int. J. Multiphase Flow 27, 1735 (2001).

[39] A. Kalaaji, B. Lopez, P. Attané, and A. Soucemarianadin, Breakup length of forced liquid jets, Phys. Fluids 15, 2469 (2003).

[40] F. J. García, H. González, J. R. Castrejón-Pita, and A. A. Castrejón-Pita, The breakup length of harmonically stimulated capillary jets, Appl. Phys. Lett. 105, 094104 (2014).

[41] J. R. Castrejon-Pita, W. R. S. Baxter, J. Morgan, S. Temple, G. D. Martin, and I. M. Hutchings, Future opportunities and challenges of inkjet technologies, Atomization and sprays 23, 541 (2013).

[42] J. C. Lasheras and E. J. Hopfinger, Liquid jet instability and atomization in a coaxial gas stream, Annu. Rev. Fluid Mech. 32, 275 (2000).

[43] R. D. Reitz, Modeling atomization processes in high-pressure vaporizing sprays, Atomisation Spray Technol. 3, 309 (1987). 
[44] W. van Hoeve, S. Gekle, J. H. Snoeijer, M. Versluis, M. P. Brenner, and D. Lohse, Breakup of diminutive Rayleigh jets, Phys. Fluids 22, 122003 (2010).

[45] J. O. Cruickshank and B. R. Munson, Viscous fluid buckling of plane and axisymmetric jets, J. Fluid Mech. 113, 221 (1981).

[46] E. Lorenceau, D. Quéré, and J. Eggers, Air Entrainment by a Viscous Jet Plunging into a Bath, Phys. Rev. Lett. 93, 254501 (2004).

[47] B. Pouligny and M. Chassande-Mottin, Air Ingestion by a Buckled Viscous Jet of Silicone Oil Impacting the Free Surface of the Same Liquid, Phys. Rev. Lett. 100, 154501 (2008).

[48] K. T. Kiger and J. H. Duncan, Air-entrainment mechanisms in plunging jets and breaking waves, Annu. Rev. Fluid Mech. 44, 563 (2012).

[49] N. B. Speirs, Z. Pan, J. Belden, and T. T. Truscott, The water entry of multi-droplet streams and jets, J. Fluid Mech. 844, 1084 (2018).

[50] J. H. Lienhard, An influence of superheat upon the spray configurations of superheated liquid jets, J. Basic Eng. 88, 685 (1966).

[51] T. Alghamdi, S. T. Thoroddsen, and J. Hernández-Sánchez, Ultra-high speed visualization of a flashboiling jet in a low-pressure environment, Int. J. Multiphase Flow 110, 238 (2019).

[52] Y. Abe, E. Matsuo, T. Arai, H. Nariai, K. Chitose, K. Koyama, and K. Itoh, Fragmentation behavior during molten material and coolant interactions, Nucl. Eng. Des. 236, 1668 (2006).

[53] A. M. Mathai, A. K. Sharma, J. Anandan, B. Malarvizhi, S. K. Das, B. Nashine, and P. Chellapandi, Investigation of fragmentation phenomena and debris bed formation during core meltdown accident in SFR using simulated experiments, Nucl. Eng. Des. 292, 87 (2015).

[54] P.-C. Chen, W.-C. Wang, W. L. Roberts, and T. Fang, Spray and atomization of diesel fuel and its alternatives from a single-hole injector using a common rail fuel injection system, Fuel 103, 850 (2013).

[55] L. Wang, J. A. Badra, W. L. Roberts, and T. Fang, Characteristics of spray from a GDI fuel injector for naphtha and surrogate fuels, Fuel 190, 113 (2017).

[56] J. Eggers and E. Villermaux, Physics of liquid jets, Rep. Prog. Phys. 71, 036601 (2008).

[57] D. H. Peregrine, G. Shoker, and A. Symon, The bifurcation of liquid bridges, J. Fluid Mech. 212, 25 (1990).

[58] X. D. Shi, M. P. Brenner, and S. R. Nagel, A cascade of structure in a drop falling from a faucet, Science 265, 219 (1994).

[59] X. Zhang and O. A. Basaran, An experimental study of dynamics of drop formation, Phys. Fluids 7, 1184 (1995).

[60] J. Eggers, Nonlinear dynamics and breakup of free-surface flows, Rev. Mod. Phys. 69, 865 (1997).

[61] J. C. Burton, J. E. Rutledge, and P. Taborek, Fluid pinch-off in superfluid and normal ${ }^{4} \mathrm{He}$, Phys. Rev. E 75, 036311 (2007).

[62] T. P. Torda, Evaporation of drops and breakup of sprays, Astronaut. Acta 18, 383 (1973).

[63] R. J. Donnelly and C. F. Barenghi, The observed properties of liquid helium at the saturated vapor pressure, J. Phys. Chem. Ref. Data 27, 1217 (1998).

[64] E. W. Lemmon, M. O. McLinden, and D. G. Friend, Thermophysical properties of fluid systems, in NIST Chemistry WebBook, NIST Standard Reference Database Number 69, edited by P. J. Linstrom and W. G. Mallard (National Institute of Standards and Technology, Gaithersburg, MD).

[65] D. O. Ortiz Vega, A New Wide Range Equation of State for Helium-4, Ph.D. thesis, Texas A\&M University, 2013.

[66] J. F. Allen and H. Jones, New phenomena connected with heat flow in helium II, Nature 141, 243 (1938).

[67] T. V. Chagovets and S. W. V. Sciver, A study of thermal counterflow using particle tracking velocimetry, Phys. Fluids 23, 107102 (2011).

[68] J. Gao, W. Guo, and W. F. Vinen, Determination of the effective kinematic viscosity for the decay of quasiclassical turbulence in superfluid ${ }^{4} \mathrm{He}$, Phys. Rev. B 94, 094502 (2016).

[69] J. S. Langer and M. E. Fisher, Intrinsic Critical Velocity of a Superfluid, Phys. Rev. Lett. 19, 560 (1967).

[70] E. Rolley, C. Guthmann, and M. Pettersen, The hydraulic jump and ripples in liquid helium, Physica B: Condensed Matter 394, 46 (2007). 
[71] H. W. Coleman and G. Steel, Experimentation, Validation, and Uncertainty Analysis for Engineers (John Wiley \& Sons, New York, 2018).

[72] See Supplemental Material at http://link.aps.org/supplemental/10.1103/PhysRevFluids.5.044001 for corresponding videos.

[73] R. F. Day, E. J. Hinch, and J. R. Lister, Self-Similar Capillary Pinchoff of an Inviscid Fluid, Phys. Rev. Lett. 80, 704 (1998).

[74] J. Eggers and T. F. Dupont, Drop formation in a one-dimensional approximation of the Navier-Stokes equation, J. Fluid Mech. 262, 205 (1994).

[75] R. W. Fenn and S. Middleman, Newtonian jet stability: The role of air resistance, AIChE J. 15, 379 (1969).

[76] Z. Wu, L. Wang, J. A. Badra, W. L. Roberts, and T. Fang, GDI fuel sprays of light naphtha, PRF95 and gasoline using a piezoelectric injector under different ambient pressures, Fuel 223, 294 (2018). 